\title{
Introduction
}

Decades of research on user modeling, personalization, and recommender systems have led to a solid body of general approaches, principles, algorithms, and tools. Personalization has become a core functionality in search engines, online stores, and social media feeds.

In the area of human-computer interaction (HCI), personalization plays a prominent role as well. For instance, interaction with computer-based devices requires users to exhibit a wide range of physical and cognitive abilities, which differ from person to person. Further, most users have their own preferred interaction styles, modalities, devices, and user interfaces, which raises the need for personalization in all aspects of HCI. Even though personalization is a commonly adopted technology, many principles and insights from the research community have not yet sufficiently been applied.

User modeling refers to the process of collecting data about users, inferring a user model in order to apply this model to customize and personalize systems. Personalized systems use this model to adapt the interaction with the users to their requirements, preferences, background knowledge, restrictions, usage contexts, and/or goals. The adaptation can be carried out in different manners, e. g., modifying a user interface according to the user's capabilities or knowledge about the system, or proposing interesting and relevant items to a user in a recommender system to reduce information overload. In this book, core researchers present the state-of-the-art research and practice of adaptation and personalization from the perspective of $\mathrm{HCI}$ in a wide range of areas.

The book chapters were elicited via a public call for chapters. We received $24 \mathrm{ab}$ stracts and accepted 11 full chapter submissions after a thorough selection and reviewing process. We have grouped the chapters into the following parts: (i) Foundations of user modeling, (ii) User input and feedback, and (iii) Personalization approaches.

\section{Foundations of user modeling}

The first chapter, “Theory-grounded user modeling for personalized HCI," by Mark P. Graus and Bruce Ferwerda, presents a literature overview of models from psychological theory that can be used in personalization. The motivation is to leverage the theoretical understanding between behavior and user traits that can be used to characterize individual users. They propose a step-by-step approach on how to design personalized systems that take users' traits into account.

Sarah Theres Völkel, Ramona Schödel, Daniel Buschek, Clemens Stachl, Quay Au, Bernd Bischl, Markus Bühner, and Heinrich Hußmann continue the foundation part in their chapter "Opportunities and challenges of utilizing personality traits for personalization in HCI.” They discuss opportunities and challenges of assessing and utiliz- 
ing personality traits in personalized interactive systems and services. The chapter includes approaches of using personality traits for recommender systems in several use cases for personality-aware personalization, such as personal communication between users.

\section{User input and feedback}

The second part of the book focuses on user input and feedback options in adaptive systems. Mirjam Augstein and Thomas Neumayr showcase personalized interaction in their chapter "Automated personalization of input methods and processes." They present a software framework that provides a template for a feasible technical infrastructure. Furthermore, the authors explain a specific case study of personalized interaction that was implemented based on the framework, and discuss an evaluation process and results for this use case.

Tobias Moebert, Jan N. Schneider, Dietmar Zoerner, Anna Tscherejkina, and Ulrike Lucke look at cause-and-effect models behind adaptive training systems in their chapter "How to use socio-emotional signals for adaptive training." They explain mechanisms for implementing the models and also empirical results from a study on the training of emotion recognition by people with autism as an example. They present two approaches; one is to extend the algorithm regarding dimensions of difficulty in social cognition, the second is to make use of socio-emotional signals of the learners in order to further adapt the training system.

"Explanations and user control in recommender systems" by Dietmar Jannach, Michael Jugovac, and Ingrid Nunes reviews explanations and feedback mechanisms in recommender systems. Often, these systems are black boxes for users and do not provide information on why items were recommended. In addition, users have frequently very limited means to control the recommendations, which may lead to limited trust and acceptance.

\section{Personalization approaches}

The third part of the book is about the application of adaptation and personalization in interactive systems in various domains. "Tourist trip recommendations - foundations, state of the art, and challenges” by Daniel Herzog, Linus W. Dietz, and Wolfgang Wörndl surveys the field of Tourist Trip Design Problems (TTDPs). TTDPs deal with the task of supporting tourists in creating personalized trips with sets or sequences of points of interest or other travel-related items. The authors present trip recommender systems with a focus on recommendation techniques, data analysis, and user interfaces. 
Continuing in the tourism domain, Wilfried Grossmann, Mete Sertkan, Julia Neidhardt, and Hannes Werthner present their chapter "Pictures as a tool for matching tourist preferences with destinations.” They introduce a so-called Seven-Factor Model for characterizing the preferences of tourists by assigning values in this model with a picture-based approach. For this purpose, users select pictures that represent various personality aspects and destination descriptions. The authors evaluated their profile acquisition method with a study using data from a travel agency.

The chapter "Towards personalized virtual reality touring through cross-object user interfaces” by Xiangdong Li, Yunzhan Zhou, Wenqian Chen, Preben Hansen, Weidong Geng, and Lingyun Sun is about real-time adaptation in virtual environments. The authors propose cross-object user interfaces for personalized virtual reality touring. The approach consists of two components: a Deep Learning algorithm-based model to predict the user's visual attention from the past eye movement patterns in order to determine which virtual objects are likely to be viewed next, and delivery mechanisms that determine what should be displayed and where and when on the user interfaces.

Music recommender systems represent a widely adopted application area for personalized systems and interfaces. In their chapter "User awareness in music recommender systems,” Peter Knees, Markus Schedl, Bruce Ferwerda, and Audrey Laplante focus on the listener's aspects of music recommender systems. The authors review different factors that influence relevance for music recommendation, for example the individual listener's background and context. This is complemented by a discussion on user-centric evaluation strategies for music recommender systems and a reflection on current barriers as well as on strategies to overcome them.

The chapter "Personalizing the user interface for people with disabilities" by Julio Abascal, Olatz Arbelaitz, Xabier Gardeazabal, Javier Muguerza, Juan E. Pérez, Xabier Valencia, and Ainhoa Yera deals with user interface personalization for people with disabilities. The authors present methods and techniques that are applied to research and practice in this important application area for personalized HCI. They outline possible approaches for diverse application fields where personalization is required, for example accessibility to the web using transcoding or personalized eGovernment.

The book concludes with the chapter "Adaptive workplace learning assistance" by Miloš Kravčík. He gives a reflective overview of the progress of adaptive workplace learning assistance and discusses three important areas of lifelong and workplace learning which correspond to basic theories of learning. The author highlights several prospective approaches of learning technology that aim to address current issues and lead to better personalization of learning experiences. 
